\title{
Aufbruchstimmung im Koalitionsvertrag
}

Mit dem Motto „Mehr Fortschritt wagen“ haben die künftigen Koalitionspartner ein klares Signal gesetzt, dass es für die anstehende Transformation die „Lust auf Zukunft“ und den „Mut zu Veränderungen“ braucht. Konsequenterweise startet der Koalitionsvertrag deshalb mit dem Anspruch, den Staat selbst zu modernisieren und dafür zu sorgen, dass umfassende öffentliche und private Investitionen in die Modernisierung des Landes getätigt werden können. Es sollen öffentliche Investitionen in Klimaschutz, Digitalisierung, Bildung und Forschung getätigt sowie Anreize für private Investitionen gesetzt und Raum für unternehmerisches Wagnis gegeben werden. Diese Themen stehen auch im Mittelpunkt des diesjährigen Jahresgutachtens des Sachverständigenrats. In der Pandemie ist der Handlungsbedarf bei der Digitalisierung besonders deutlich geworden. Handlungsbedarf gibt es aber auch bei den notwendigen Schritten hin zur Klimaneutralität, etwa beim Ausbau der erneuerbaren Energien, sowie generell bei der Durchführung von Infrastrukturmaßnahmen. Deshalb ist es nur folgerichtig, dass der Koalitionsvertrag die Modernisierung des Staates an den Anfang stellt und fordert, der Staat solle selbst agiler und digitaler werden.

Im Zentrum des Vorhabens, den Staat zu modernisieren, steht deshalb die Digitalisierung der Verwaltung. Proaktives Verwaltungshandeln durch antragslose und automatisierte Verfahren soll gesetzlich verankert werden. Richtig sind auch die Feststellung, dass Modernisierung "nur mit einem starken öffentlichen Dienst“ gelingt, und die Festlegung darauf, Digitalisierung zum Kernbestandteil der Ausbildung der öffentlichen Beschäftigten zu machen. Dies sollte - wird aber im Vertrag nicht explizit genannt - auch für die Fortbildung der öffentlichen Beschäftigten gelten. Laut Vertrag sollen die Kompetenzen für Digitalisierung in der Bundesregierung neu geordnet und gebündelt werden. Auch soll es ein zentrales zusätzliches Digitalbudget geben. Das ist grundsätzlich zu befürworten, aber es bleibt unklar, ob dieses Budget durch das Ministerium für Verkehr und Digitales verantwortet werden soll. Die Beschleunigung von (Infrastruktur-)Maßnahmen soll durch einen „Pakt für Planungs-, Genehmigungs- und Umsatzbeschleunigung mit den Ländern" vorangetrieben werden. Zum Anspruch der Beschleunigung passt auch, dass die Digitalisierung von Planungs- und Genehmigungsprozessen priorisiert umgesetzt, die Behörden mit der notwendigen Technik ausgestattet sowie die IT-Schnittstellen zwischen Bund und Ländern standardisiert werden sollen.

Eine Beschleunigung der Verfahren ist notwendig, um den Ausbau erneuerbarer Energien in Deutschland voranzutreiben. Ohne sie wird das im Vertrag gesetzte Ziel, bis $203080 \%$ des Strombedarfs aus erneuerbaren Energien zu decken, nicht einzuhalten sein. Um die Verfahren abzukürzen, sollen künftig „,bei Planänderungen nach Bürgerbeteiligung nur noch neu Betroffene" beteiligt werden und „Einwendungen nur mehr gegen Planänderungen zulässig“ sein. Geplant ist auch: „Für unsere gemeinsame Mission, die Planung von Infrastrukturprojekten, insbesondere den Ausbau der erneuerbaren Energien drastisch zu beschleunigen, wollen wir das Verhältnis von Klimaschutz und Artenschutz klären." Zudem wird konkretisiert, wie diese Klärung aussehen soll: Bei der Abwägung von Schutzgütern soll es einen zeitlich bis zum Erreichen der Klimaneutralität befristeten Vorrang für erneuerbare Energien geben. 
Gemäß der Tatsache, dass der Klimaschutz globale Anstrengungen braucht, unterstützt die Koalition die Gründung eines Klimaclubs und die Einführung eines europaweiten $\mathrm{CO}_{2}$-Grenzausgleichsmechanismus, der WTO-konform ist und die Exportindustrie nicht benachteiligt. Richtige Ziele, die aber, wie im Jahresgutachten diskutiert, nicht einfach umzusetzen sein werden.

Die Koalition bekennt sich zu dauerhaft hohen Investitionszusagen, die in einem langfristigen Investitionsplan dargelegt werden sollen. Wie wir im Jahresgutachten ausgeführt haben, ist eine solche Verstetigung der Investitionen wichtig, um für Planungssicherheit und den notwendigen Kapazitätsaufbau zu sorgen, ohne den der Mittelabfluss nicht gewährleistet werden kann, ein Kapazitätsaufbau im privaten (z.B. Bauindustrie) wie auch im öffentlichen Sektor (Planung und Umsetzung). Zur Finanzierung dieser Investitionen im Rahmen der Schuldenbremse werden im Koalitionsvertrag unterschiedliche Optionen angesprochen, wie z. B. die kapitalmarktnahe Unterstützung privater Investitionen durch die KfW, die zur Innovations- und Investitionsagentur umgebaut werden soll, die Stärkung der Investitionen öffentlicher Unternehmen wie der Bahn oder der Bundesanstalt für Immobilienaufgaben durch Kreditermächtigungen und Eigenkapitalstärkung, bei gleichzeitiger Priorisierung der Ausgaben und des Abbaus umweltschädlicher Investitionen. Die Vor- und Nachteile dieser und anderer Optionen hatten wir im Jahresgutachten zur Diskussion gestellt.

Zu begrüßen ist, dass die Koalition gemeinsam mit den Ländern die öffentlichen Bildungsausgaben deutlich steigern will. In der Pandemie sind erhebliche Lern- und Entwicklungsdefizite bei den Kindern und Jugendlichen entstanden, bei Kindern aus sozial benachteiligten Verhältnissen noch stärker als bei anderen. Wie wir im Jahresgutachten berichten, verschärfte sich so die im internationalen Vergleich ohnehin große soziale Kluft im Bildungserfolg. Nachdem 2019 das grundgesetzlich verankerte Kooperationsverbot aufgehoben wurde, spricht die Koalition jetzt von einem Kooperationsgebot und verspricht ein Jahrzehnt der Bildungschancen. Passend dazu liegt der Fokus auf frühkindlicher Bildung, der Förderung sozial benachteiligter Schulen und einem grundlegend reformierten BAföG, aber auch auf Lehrerfortbildung mit Schwerpunkten in der digitalen Bildung. Eine Stärkung der Transparenz der Lernerfolge in den einzelnen Bundesländern, wie wir im Jahresgutachten angemahnt hatten, findet sich im Koalitionsvertrag leider nicht.

Zudem steht die Koalition auch vor der Aufgabe, die Folgen des demografischen Wandels zu bewältigen. Sie strebt richtigerweise an, die Erwerbsbeteiligung von Frauen zu erhöhen. Die oftmals - auch im Jahresgutachten - dargestellten negativen Anreizwirkungen des Ehegattensplittings werden aber nicht erwähnt. Es ist zu hoffen, dass die bekundete Absicht, die Familienbesteuerung weiterzuentwickeln, die Tür für eine Reform offen hält. Immerhin soll, wie auch vom Sachverständigenrat vorgeschlagen, die Kombination aus den Steuerklassen III und V in das Faktorverfahren der Steuerklasse IV überführt werden. Auch wenn im Koalitionsvertrag betont wird, dass Minijobs nicht zur Teilzeitfalle speziell für Frauen werden sollen, sind sie aktuell genau das und es wird leider im Vertrag nicht erkennbar, wie dies künftig verhindert werden soll. Selbst wenn es gelingen sollte, die Erwerbsbeteiligung von Frauen und die Nettoeinwanderung zu steigern, ist zweifelhaft, ob dies ausreichen wird, um die Nachhaltigkeit des Rentensystems zu sichern. Der Vertrag hält an den bisherigen Haltelinien fest, eine Anhebung des gesetzlichen Rentenalters wird es nicht geben. Wie die Nachhaltigkeit der Rentenfinanzierung gelingen soll, bleibt daher unklar. Daran ändert auch der begrüßenswerte Einstieg in einen kapitalgedeckten Teil der gesetzlichen Rente wenig. Immerhin: Der Nachholfaktor wurde wieder eingesetzt und damit vermieden, dass die Rentenerhöhungen höher ausfallen als die pandemiebedingt niedrigeren Lohnsteigerungen. 